\title{
EDITORIAL
}

\section{Best practice in high-performance sport}

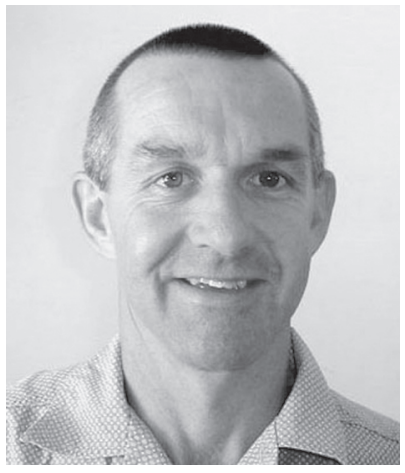

The South African Sports Medicine Association (SASMA) is undergoing a renaissance with a realignment of their vision and a drive to boost membership. The necessity of having a strong sports medicine association is probably more important now than ever before. The sad deterioration of the standard of sport in South Africa and the attempt by some politicians and administrators to hijack sport to fulfill their own agendas is at a critical level.

The contribution of SASMA to ensure that the situation does not spiral out of control needs to be clarified. Among the many goals of SASMA, perhaps one of the most important is to provide a vehicle for continuing education so that the members are able to maintain a high level of service provision to patients, athletes and coaches. This high level of service needs to adhere to the principles of best practice. This is perhaps easier said than done. For starters, what constitutes best practice when dealing with high-performance elite teams? This question was raised and discussed on numerous occasions at the recent Sports Medicine Conference in Cape Town. While there was general agreement that the term 'best practice' described a procedure or method that consistently resulted in the desired response, there was less agreement on how this could be achieved. Some delegates put forward the view that best practice was synonymous with the concept of evidence-based practice, which in turn strives to apply information gained from scientific experimentation. Most trained scientists would concur with this view, while at the same time expressing concern that the scientific process needs to be interpreted and applied accurately. Failing this, the system crumbles.

While in a clinical setting the inappropriate use of various medical treatments may have life-threatening consequences, the same does not apply to the application of various modalities for training and recovery in sport. Therefore there is less scrutiny of these methods and a general muddling of what is based on the personal experience of a practitioner versus what can be considered best practice as a result of supporting scientific evidence. Consider the practice of recovery after training and competition - inadequate recovery is listed as one of the major obstacles in attaining optimal performance. ${ }^{2}$ While many researchers have attempted to define the ideal recovery strategy, ${ }^{1}$ the research on recovery strategies is limited for the following reasons: (i) often untrained people are exposed to a gruelling exercise protocol and then studied with the expectation that they will respond similarly to elite athletes - we know this is not the case; (ii) the protocols used to induce fatigue are not sufficiently specific to the demands of a sport; and (iii) the markers of recovery used in research (i.e. creatine kinase activity in the blood, pain, performance) are not necessarily valid markers of recovery.

Furthermore, the types of experiments which have a high believability factor are the randomised double-blind placebocontrolled trials. Unfortunately these trials are almost impossible to conduct with elite professional athletes. For these reasons the body of scientific information which can be applied to the management of athletes and players after training and competition is limited. This has led to the support staff placing a greater emphasis on personal preferences and experiences of their colleagues when deciding on best practice principles. Under these circumstances there is no alternative. However, there should be extra vigilance when these practices are used. Consider for example the use of cold-water immersion of players after training and competition. This practice is based in the theory that inflammation which occurs after hard training and competition is a negative biological consequence and therefore should be prevented - while this logic may be correct when used to treat injured muscle and soft tissue in the acute phase of injury, does it really apply to training-induced stress? A recently published study would suggest not. $^{3}$ This study examined the training-induced adaptations in a group of subjects who had their trained limbs exposed to cold-water immersion after every training session - surprisingly, this treatment reduced the training-induced adaptations, leading the authors to conclude that the muscle hyperthermia associated with training, and which is blocked by exposure to cold-water immersion, is an important biological stimulus for inducing training adaptations. This is just an example of how treatments may be misused in the absence of good convincing data. It is unlikely that this will change in the foreseeable future. Therefore there is no simple solution other than encouraging practitioners and service providers working with sports participants to have open minds and communicate with colleagues, be alert for dogmatic viewpoints, and be aware of pseudo-science being used to promote extreme viewpoints. SASMA can assist in this ongoing education role providing the regions become strong and vibrant.

\section{Mike Lambert \\ Editor-in-Chief}

1. Barnett A. Using recovery modalities between training sessions in elite athletes: does it help? Sports Med 2006; 36: 781-96.

2. Smith DJ. A framework for understanding the training process leading to elite performance. Sports Med 2003; 33: 1103-26.

3. Yamane M, Teruya H, Nakano M, Ogai R, Ohnishi N, Kosaka M. Postexercise leg and forearm flexor muscle cooling in humans attenuates endurance and resistance training effects on muscle performance and on circulatory adaptation. Eur J Appl Physiol 2006; 96: 572-80. 